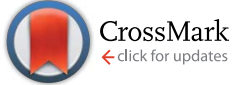

Cite this: RSC Adv., 2015, 5, 24528

Received 17th November 2014 Accepted 24th February 2015

DOI: 10.1039/c4ra14693j

www.rsc.org/advances

\section{Catalyst-free synthesis of carbon nanospheres for potential biomedical applications: waste to wealth approach $\dagger$}

\author{
Shoriya Aruni Abdul Manaf, ${ }^{a}$ Partha Roy, ${ }^{\mathrm{b}}$ Korada V. Sharma, ${ }^{\mathrm{C}}$ Zainab Ngaini, ${ }^{\mathrm{d}}$ \\ Victor Malgras, ${ }^{e}$ Ali Aldalbahi, ${ }^{f}$ Saad M. Alshehri, ${ }^{f}$ Yusuke Yamauchi ${ }^{\mathrm{e}}$ \\ and Gurumurthy Hegde*ag
}

A single step and simple pyrolysis technique is used to prepare carbon nanospheres (CNSs) from natural biowaste sago hampas in a nitrogen atmosphere without any catalyst. Scanning electron microscope (SEM) images along with transmission electron microscope (TEM) images show evidence of high quality CNSs with a good particle size uniformity. Both X-ray diffraction (XRD) and Raman data show the presence of graphitic characteristic peaks of CNSs. Zeta-potential study reveals that the obtained CNSs can be well dispersed in solution making them suitable for cell imaging applications. The use of biowaste sago hampas is very important from the viewpoint of sustainable synthesis of functional CNSs for the future.

\section{Introduction}

Carbon nanomaterials with different shapes and structures have attracted widespread attention from many researchers working in various fields around the globe due to their vast applications in diverse fields such as catalyst supports, ${ }^{\mathbf{1}}$ anode materials of Li-ion batteries, ${ }^{2}$ nanostructures in diagnosis, ${ }^{3}$ and multiphoton bioimaging. ${ }^{4}$ For imaging and efficient drug delivery, various carbon-based nanomaterials have been used as drug delivery vehicles, often tagged with some fluorescent agents and antibodies. ${ }^{5-7}$ Different forms of carbon nanostructures, including carbon nanotubes, ${ }^{\mathbf{8}, 9}$ carbon nanofibers, ${ }^{10,11}$ carbon trees, ${ }^{12,13}$ carbon spheres, ${ }^{14,15}$ mesoporous carbons, ${ }^{16}$ carbon capsules, ${ }^{17}$ and carbon nanowalls ${ }^{18}$ have been developed since the last 20 years. Among various shapes, carbon nanospheres (CNSs) are gaining interest as catalyst supports,

${ }^{a}$ Faculty of Industrial Sciences and Technology, Universiti Malaysia Pahang, 26300 Gambang, Kuantan, Malaysia.E-mail: murthyhegde@gmail.com

${ }^{b}$ Faculty of Engineering Technology, Universiti Malaysia Pahang, 26300 Gambang, Kuantan, Malaysia

${ }^{c}$ Department of Mechanical Engineering, Faculty of Engineering, University Technology PETRONAS, Bandar Seri Iskandar, 31750, Tronoh, Perak, Malaysia

${ }^{d}$ Department of Chemistry, Faculty of Resource Science and Technology, Universiti Malaysia Sarawak, 94300 Sarawak, Malaysia

${ }^{e}$ World Premier International (WPI) Research Center for Materials Nanoarchitectonics (MANA), National Institute for Materials Science (NIMS), 1-1 Namiki, Tsukuba, Ibaraki 305-0044, Japan

${ }^{f}$ Department of Chemistry, College of Science, King Saud University, Riyadh 11451, Saudi Arabia

${ }^{g}$ BMS $R$ and D Centre, BMSCE, Bull Temple Road, 560019 Bangalore, India

$\uparrow$ Electronic supplementary information (ESI) available: See DOI: $10.1039 / \mathrm{c} 4 \mathrm{ra} 14693 \mathrm{j}$ fillers for polymer nanocomposites, column packing and lubricating materials, biomedical drug carriers, etc. In recent reports, the CNSs also exhibited excellent photoluminescent properties. ${ }^{19-21}$

Various synthetic approaches have been reported to prepare CNSs. $^{22-25}$ All of the methods for carbonization involve the use of either a template, a catalyst, or an oxidising agent. The templating methods require time-consuming process to collect only pure CNSs by separating the material from the used catalysts. Graphite powders, petroleum pitch, carbon rich polymers, and other kinds of liquid/gaseous hydrocarbons have been extensively used for the synthesis of CNSs as precursor. As these chemicals becoming scarce resources and have detrimental impacts on the environment, the use of alternative carbon sources for the synthesis of carbonaceous materials is in high demand in various fields. Thus, the carbon source and the synthetic method are the two key issues for the development of environment-friendly and cost-effective preparation of CNSs. Plant biomasses, biobased oils, and hydrocarbons may serve as the best alternative and renewable carbon sources. Very less effort has been made in this regard to prepare CNSs, ${ }^{26,27}$ although the biomasses have been utilized for the synthesis of chemicals through green routes. ${ }^{28}$

This encouraged us to search for new carbon sources and very recently we reported CNSs from oil palm leaves and its electrochemical applications. ${ }^{29}$ Following the urge to find new carbon sources for the preparation of CNSs, herein we propose the use of bio-waste sago 'hampas' which is an inexpensive fibrous residue obtained from sago palm tree. Sago hampas are rich in cellulose and hemicelluloses with small percentage of lignin content and have a porous structure. ${ }^{27 b, 30}$ The porous nature of sago hampas prompted us to prepare new CNSs by a 